\title{
Evolución inusual de fibroadenomas mamarios múltiples en adolescente con metrorragia disfuncional
}

\author{
Marcela Menéndez A. ${ }^{1}$, Claudio Berríos G. ${ }^{2}$ Claudia Zajer A. ${ }^{1}$, Carolina Pastene $S .{ }^{1}$ \\ ${ }^{1}$ Unidad de Ginecología Infanto-Juvenil, División de Obstetricia y Ginecología; ${ }^{2}$ Departamento de Radiología. Escuela \\ de Medicina, Pontificia Universidad Católica de Chile.
}

\section{RESUMEN}

Se describe la inusual evolución de fibroadenomas (FA) mamarios múltiples en una adolescente de 11 años en tratamiento con progestinas cíclicas por metrorragia disfuncional. Inicialmente la ecografía mamaria mostró más de 10 lesiones sólidas en cada mama de hasta $2,6 \mathrm{~cm}$, compatibles con fibroadenomas. Al cabo de 13 meses las lesiones se redujeron en número y tamaño. Se discute la historia natural de los FA, la influencia hormonal en la etiología de los FA y finalmente el diagnóstico ecográfico y manejo de este cuadro en la adolescencia. Se plantea, a modo de hipótesis, que existe un desbalance estrógeno-progesterona (E-P) dado por ciclos anovulatorios en el periodo posmenárquico, que se manifestó con metrorragia disfuncional y la presencia de FA. Al administrar progesterona cíclica se reequilibra la relación E-P explicando el control del ciclo y la involución de las lesiones mamarias.

\section{PALABRAS CLAVE: Fibroadenoma, tumor mamario, adolescencia}

\section{SUMMARY}

We describe the unusual evolution of multiple fibroadenomas $(F A)$ in a 11-years old adolescent during treatment of an abnormal uterine bleeding with cyclic progestins. Inicially ultrasound demonstrated more than 10 masses in each breast, up to $2,6 \mathrm{~cm}$ diameter, compatible with fibroadenomas. After 13 month follow up lesions decreased in number and size. We discuss the natural history of FA, the hormonal influence in FA etiology, the ultrasound diagnosis and the managment of this pathology in adolescents. We hypothesized that there is an estrogen/progestin imbalance, due to the anovulatory cycles of the post menarche period that caused the AUB and FA. Cyclic progesterone balanced the E/P relation, thus managing both AUB and causing regression of the breast masses.

\section{KEY WORDS: Fibroadenoma, breast tumor, adolescence}

\section{INTRODUCCIÓN}

Los fibroadenomas (FA) son tumores fibroepiteliales benignos de la mama y corresponden alrededor del $90 \%$ de las lesiones sólidas de la mama en la adolescencia (1). Su incidencia es desconocida, estimándose su prevalencia en $3,25 \%$ en una serie de adolescentes (2). EI FA se desarrolla a partir de las diferentes estructuras lobulares de la mama. El desarrollo lobular es máximo durante la adolescencia lo que explica la frecuencia de este tumor a esta edad (3). De acuerdo a la clasificación de ANDI 
(Aberrations In the Normal Development and Involution of the Breast): los pequeños FA serían normales, los clínicamente detectables una leve aberración de un proceso normal y los FA gigantes o múltiples estarían al final del espectro, considerándolos patológicos (4).

Aparecen habitualmente en la adolescencia tardía pero pueden encontrarse hasta 1 a 2 años antes de la menarquia (1). Clínicamente se presentan como lesiones bien circunscritas palpables, elásticas y móviles, más frecuentemente ubicadas en el cuadrante superoexterno de la mama. Su tamaño habitual fluctúa entre 1 a $3 \mathrm{~cm}$. El concepto de FA gigante se reserva para lesiones mayores a los $5 \mathrm{~cm}$ (1). Generalmente son únicos, pero los $F A$ múltiples (más de 3 a 5 lesiones por mama según diferentes autores) se describen en 15 a $20 \%$ de estas pacientes, siendo raros los casos con más de 5 lesiones por mama (5).

Del punto de vista histológico el FA es el resultado de una exageración del desarrollo normal de un lóbulo, no existiendo diferencia entre un FA y un lóbulo hiperplásico (6).

La ecografía es el examen de elección para el diagnóstico diferencial de masas mamarias en la adolescente. Dado la cantidad de tejido fibroso a esta edad la mamografía no es de utilidad (7).

El manejo es controversial, sin embargo la mayor parte de los autores recomienda el control ecográfico, reservando la biopsia y/o extirpación para las lesiones grandes, atípicas o de rápido crecimiento $(1,7,8)$.

El objetivo de esta comunicación es discutir la etiología y el manejo de los fibroadenomas múltiples en la adolescencia a partir de un caso clínico.

\section{Caso clínico}

Se presenta el caso de una paciente de 11 años derivada a la consulta de ginecología infanto-juvenil de la Pontificia Universidad Católica de Chile por una metrorragia disfuncional asociada una probable fibroadenomatosis mamaria múltiple. Tiene el antecedente de menarquia precoz (9 años 7 meses), cursó inicialmente con oligomenorrea seguida por una metrorragia disfuncional que fue tratada con anticonceptivos orales (etinilestradiol $30 \mathrm{mcg}$ asociado a levonorgestrel $150 \mathrm{mcg}$, luego desogestrel $150 \mathrm{mcg}$ ) por 10 meses. Se suspendieron los anticonceptivos diez días antes del ingreso por la presencia de múltiples lesiones sólidas mamarias en la ecografía (más de 10 en cada mama).
Al momento de la consulta presenta un flujo rojo abundante de 10 días de evolución. No refiere otros antecedentes mórbidos relevantes, no tiene de sangrado de mucosas (epistaxis o gingivorragia). Adenoidectomía sin complicaciones. Madre con hipotiroidismo y abuela materna diabética tipo II. No hay antecedentes de cáncer de mama ni trombosis en la familia.

Al examen físico general: talla de $158 \mathrm{~cm}$, peso 52 kg (IMC: 20,8), sin acné ni hirsutismo, tiroides normal, genitales externos sanos. En el examen mamario destaca un nódulo ovalado de $2 \mathrm{~cm}$ en cuadrante inferointerno de la mama derecha, móvil, elástico. El resto del tejido mamario es denso y nodular (sin identificarse claramente otros nódulos.) No presenta galactorrea.

Se plantea el diagnóstico de fibroadenomatosis múltiple y metrorragia disfuncional por probable anovulación. Para el estudio de los nódulos mamarios se solicita una nueva ecografía mamaria con radiólogo pediátrico y se deriva a un especialista en patología mamaria, ambos de nuestra institución. En relación a la metrorragia se decide evitar terapia estrogénica por ser los fibroadenomas estrógeno dependientes. Se indica inicialmente ácido tranexámico $500 \mathrm{mg}$ cada $8 \mathrm{hrs}$ por 5 días y progesterona micronizada $200 \mathrm{mg}$ al día por 10 días al mes a fecha fija y se deriva a hematología.

Inicialmente evoluciona con insuficiente respuesta al ácido tranexámico y la progesterona micronizada, presentando sangrados frecuentes. Se cambia el progestágeno a Didrogesterona $20 \mathrm{mg}$ al día por 12 días al mes, manteniendo el ácido tranexámico los primeros 3 a 5 días de menstruación, con lo que se logran ciclos de características normales, de 6 días de duración. Al cabo de 9 meses se cambia nuevamente el progestágeno a Acetato de Nomegestrol $5 \mathrm{mg}$ al día, 12 días al mes (este progestágeno era de elección por ser potente a nivel endometrial pero estuvo discontinuado en farmacias en esa oportunidad). Al cabo de 15 meses con progestágenos, se suspende el tratamiento siguiendo con ciclos normales, abundantes los primeros tres días.

Se realizó un estudio para determinar la etiología de la metrorragia y su repercusión a nivel del hemograma. La evaluación hormonal resultó normal (Tabla I), presentó una anemia hipocrómica, microcítica leve (hemoglobina $10,7 \mathrm{~g} / \mathrm{dL}$ ) y el estudio hematológico mostró una hiperfibrinolisis leve que explica en parte la abundancia de las menstruaciones, pero no la irregularidad. 
Tabla I

EVALUACIÓN HORMONAL DE LA PACIENTE

\begin{tabular}{lrr}
\hline Examen / unidad & Resultado & $\begin{array}{r}\text { Rango de } \\
\text { referencia }\end{array}$ \\
\hline TSH & $2,44 \mathrm{uUl} / \mathrm{ml}$ & $0,7-5,7$ \\
T4libre & $1,21 \mathrm{mg} / \mathrm{dl}$ & $0,8-2$ \\
FSH & $2,9 \mathrm{mUl} / \mathrm{ml}$ & $2,5-10,2$ \\
Pool de prolactina & $10,3 \mathrm{ng} / \mathrm{ml}$ & $3-14,4$ \\
(promedio) & & \\
Testosterona total & $12,4 \mathrm{ng} / \mathrm{dl}$ & $4,6-38,3$ \\
SHBG & $55,5 \mathrm{nmol} / \mathrm{L}$ & $24,6-122$ \\
Índice de andrógenos & 0,77 & $<4,5$ \\
libres & & \\
\hline
\end{tabular}

En relación a las lesiones mamarias la paciente fue evaluada por médico especialista en patología mamaria, quien, por la edad y las características de las lesiones, decide sólo controlar su evolución de manera ecográfica. La primera ecografía realizada muestra abundante tejido fibroglandular en ambas mamas y múltiples imágenes nodulares, al menos 10 a cada lado, de aspecto hipoecogénico y sólido, de estructura interna heterogénea, bien delimitadas, la mayor de las cuales se encuentra ubicada a derecha, a la hora 6 y alcanza aproximadamente $2,1 \times 2,6 \mathrm{~cm}$ en sus diámetros mayores. El estudio Doppler-color no mostró flujo vascular significativo. La impresión diagnóstica del radiólogo es que estas imágenes pueden corresponder a fibroadenomas múltiples.

La siguiente ecografía, realizada 6 meses después, muestra una disminución del número de imágenes nodulares encontrándose una lesión a izquierda de $0,8 \mathrm{~cm}$ y dos lesiones a derecha (una pequeña de $0,5 \mathrm{~cm}$ y otra de de 2,3 x 0,8 cm). En la tercera ecografía, realizada 6 meses después (12 meses desde el ingreso), se observan nuevamente 3 lesiones, 1 a izquierda y 2 a derecha, de 0,6 $\times 0,4$ $\mathrm{cm}, 0,7 \times 0,3 \mathrm{~cm}$ y $0,9 \times 0,3 \mathrm{~cm}$ (Figura $1 \mathrm{a}-3 \mathrm{~b}$ ). Clínicamente se observa una notoria disminución de las lesiones en particular de la que se palpaba con claridad al ingreso.

\section{DISCUSIÓN}

Tanto la cantidad de lesiones en cada mama (más de veinte en total) en nuestra paciente como la involución rápidas de estas es un hecho poco frecuente. En torno a este caso existen tres puntos interesantes a discutir: 1. la inusual evolución de las lesiones sólidas mamarias, 2. el posible efecto de los estrógenos y la progesterona en esta evolución y 3. la especificidad de la ecografía en el diagnóstico de los FA en la adolescencia.

La historia natural de los FA presenta un periodo inicial de crecimiento, pudiendo doblar el tamaño en 6 a 12 meses hasta un tamaño de 2 a 3 $\mathrm{cm}$, luego un periodo de estabilización (1). Un 5\% puede tener un crecimiento rápido, debiéndose sospechar un FA celular o juvenil o incluso un tumor phyllodes (sobre todo si el tamaño sobrepasa los $5 \mathrm{~cm}$ ), por lo que en estos casos está indicada la extirpación quirúrgica (10).

En el FA la proliferación del tejido epitelial y conectivo es armónica, igual que en la glándula normal. Con el tiempo se produce una esclerosis del tejido conectivo intralobular y el epitelio tiende a desaparecer. Esto podría explicar la disminución con el tiempo de la hormono dependencia de los FA y eventualmente de la regresión de estos (3). Sobre el $10 \%$ puede regresar en forma espontánea (11), llegando al $38 \%$ en series seguidas a mediano plazo (7 a 9 años) (12) y entre 16 a $59 \%$ de los casos en seguimientos de hasta más de 29 años (9).

El rol de la progesterona, los estrógenos y sus receptores en el desarrollo de la patología mamaria benigna es incierto. El hecho que los FA aparezcan en el periodo posmenárquico, tiendan a aumentar en el embarazo y a disminuir en la menopausia apoya el hecho que son hormono dependientes. Receptores de estrógenos y progesterona pueden encontrarse tanto en el citoplasma como en el núcleo en los FA. En un estudio molecular se encontró que en el $72 \%$ de los fibroadenomas encontrados estaba presente al menos un receptor a estrógeno (19\% ER+) o a progesterona (71\% PR+) (13).

Altos niveles de receptores a estrógenos se han correlacionado con proliferación epitelial y bajos niveles con proliferación estromal. Sin embargo el estudio del efecto de la progesterona sobre la proliferación celular ha mostrado resultados contradictorios; Mauvais-Jarvis y cols (14), Gompel y cols (15) reportaron un efecto anti-proliferativo de la progesterona sobre células de tejido mamario normal. Kramer y cols describieron que el efecto de la progesterona podía ser tanto una estimulación como una inhibición de la proliferación celular (16). También se han descrito receptores para prolactina y otros factores locales (17).

El efecto de los anticonceptivos orales (ACO) sobre la patología mamaria benigna también ha sido estudiado. No hay evidencia que el uso de ACO aumente su incidencia y algunos datos epidemiológicos podrían incluso mostrar una disminución. El componente progestagénico de los ACO podría tener un rol 

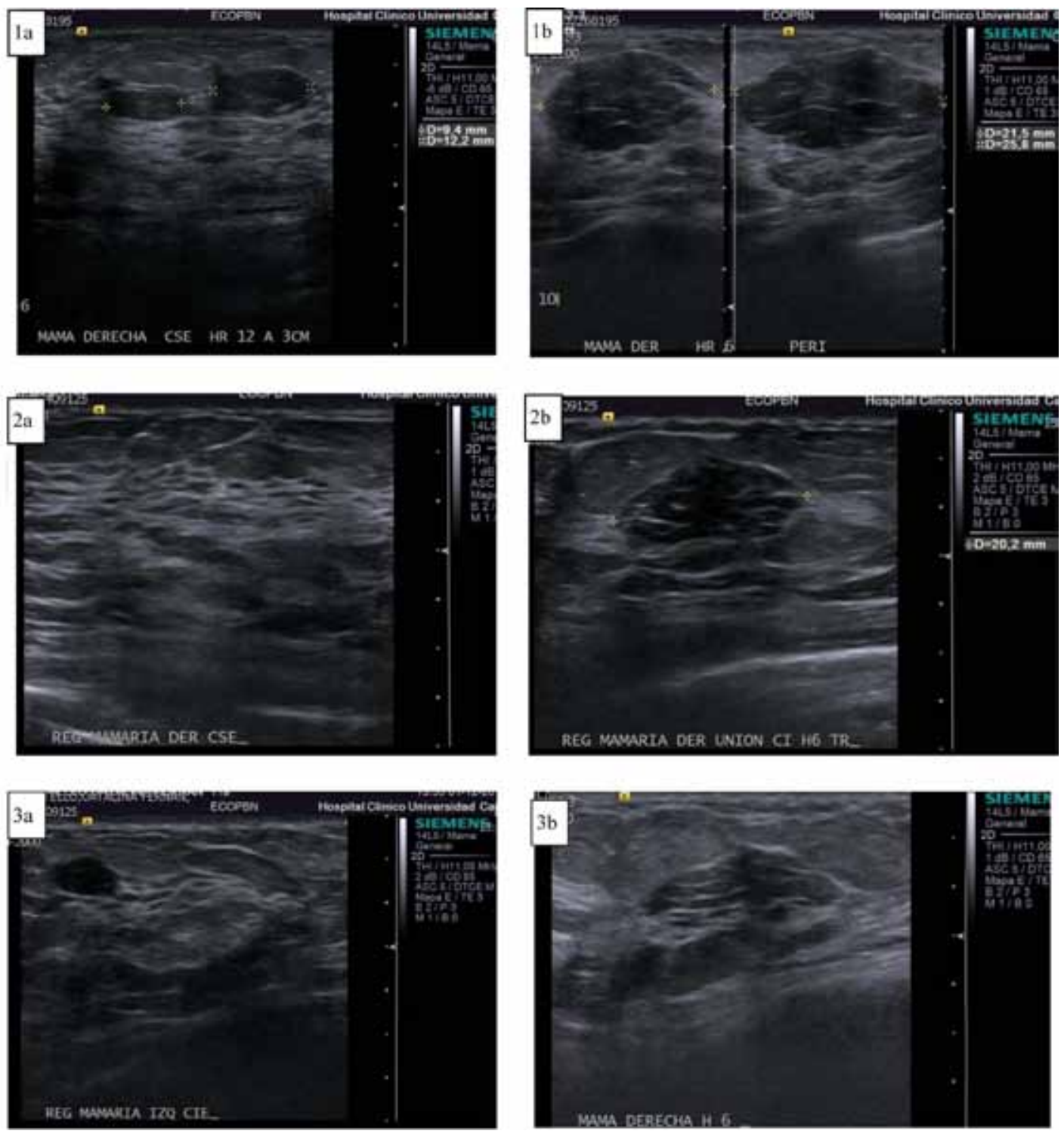

Figura 1. Algunas imágenes de ecografías mamarias (al ingreso: 1a-1b, a los 6 meses: 2a-2b, $y$ a los 12 meses posingreso: 3a-3b).

protector. En un gran estudio de cohorte de 265.402 mujeres, se evaluaron diferentes factores de riesgo para presentar fibroadenomas: el mayor tiempo de uso de ACO se relacionó con una disminución del riesgo de presentar FA (18). La edad de la menarquia no mostró relación con el riesgo de presentar $F A$, punto interesante ya que nuestra paciente presentó una menarquia precoz (18).
En la década del 80-90 se propusieron tratamientos médicos para la patología mamaria benigna. Se publicó el uso del Danazol y del Tamoxifeno en patología benigna de la mama, pero se discontinuaron por sus efectos secundarios $(19,20)$.

Para algunos autores el FA se produce por un desbalance estrógeno/progesterona (3). Basado en esto, en particular en Francia, algunos clínicos 
utilizan progestágenos derivados de la 19 nortestosterona por 15 a 20 días por ciclo con buenos resultados clínicos y ecográficos (3). En el caso de nuestra paciente, el estudio de su metrorragia disfuncional así como su edad, orientan a una anovulación funcional del periodo posmenárquico, con la consecuente falta de progesterona. No conocemos el tiempo de evolución de sus lesiones mamarias antes del ingreso ni tampoco su evolución durante el periodo de uso de los ACO. Creemos que el uso de progesterona cíclica pudo jugar un rol importante en la resolución de las lesiones, como ha sido descrito en la evolución de la patología mamaria benigna, sin embargo, no podemos descartar que se trate de su evolución natural.

Nos interesa además discutir el rol de la ecografía en el diagnóstico de los FA. En el caso presentado la sospecha diagnóstica se basó en las características clínicas y ecográficas de los nódulos dado que no se realizó biopsia. El primer punto importante es destacar que la patología mamaria maligna en la adolescencia es extremadamente rara, correspondiendo a $<1 \%$ de cáncer infantil y $<0,1 \%$ de los cáncer de mama (7). En un estudio de Smith y Burrows, el $99,7 \%$ de las biopsias fueron benignas en pacientes jóvenes menores de 25 años en las que se sospechó un fibroadenoma al examen físico o a la ecografía (21). Las características ecográficas de los fibroadenomas en adolescentes se presentan en la Tabla II, su descripción no se diferencia de la mujer adulta. Por lo tanto si la apariencia sonográfica es clásica y no presenta crecimiento rápido, la recomendación es realizar un seguimiento ecográfico, como lo hicimos con nuestra paciente $(7,22)$.

\section{CONCLUSIÓN}

Los FA múltiples en la adolescencia son poco frecuente, pero están probablemente subdiagnosticados. No existe literatura que describa la evolución natural de estas lesiones múltiples en este grupo etario. El caso presentado es poco común, tanto por el número de lesiones como por la involución rápida de ellas. Se ha reportado la involución de fibroadenomas pero a mayor plazo.

Existe controversia sobre la influencia hormonal en la etiología de los FA. Se ha planteado la importancia del estímulo estrogénico asociado a un déficit de progesterona, así como el rol de la prolactina, factores locales y características de los receptores hormonales a nivel del tejido mamario. La hipótesis por nosotros planteada en nuestra paciente es que existe un desbalance estrógeno-progesterona (E-P) dado por ciclos anovulatorios en el periodo

\section{Tabla II \\ CARACTERÍSTICAS ECOGRÁFICAS DE LOS FIBROADENOMAS EN LA POBLACIÓN PEDIÁTRICA ( $\left.{ }^{*}\right)$}

\begin{tabular}{llc}
\hline $\begin{array}{l}\text { Hallazgos } \\
\text { ecográficos }\end{array}$ & Características & $\begin{array}{c}\text { Frecuencia } \\
(\%)\end{array}$ \\
\hline Forma & Ovalada & 100 \\
Orientación & Paralelo a fibras & 100 \\
Márgenes & Delimitado & 41 \\
& Mal delimitado: & 57 \\
& -Lobulado & 2 \\
Límites & -Angular & 100 \\
Patrón ecográfico & Hipoecogénico & 84 \\
& Interfase abrupta & 2 \\
& Heterogéneo & 14 \\
& Refuerzo acústico & 98 \\
& Sombra acústica & 1 \\
Doppler color & Con flujo vascular & 81 \\
& Sin flujo vascular & 19 \\
& & \\
\hline
\end{tabular}

(*) Adaptada de: Sanchez R, Ladino-Torres MF, Bernat JA, Joe A, DiPietro MA. Breast fibroadenomas in the pediatric population: common and uncommon sonographic findings. Pediat Radiol 2010;40:1681-9.

posmenárquico, que se manifestó con una metrorragia disfuncional y la presencia de FA. Al administrar progesterona cíclica se reequilibra la relación E-P explicando el control del ciclo y la involución de las lesiones mamarias. Sin embargo esta hipótesis debe ser corroborada por estudios idealmente prospectivos de adolescentes con fibroadenomas múltiples. Finalmente creemos que en la adolescencia, edad en la que las lesiones mamarias malignas son muy poco frecuentes, el diagnóstico ecográfico de los fibroadenomas es suficientemente específico como para tener una conducta conservadora, con seguimiento ecográfico y reservar la extirpación quirúrgica y biopsias para las lesiones de crecimiento rápido, grandes o atípicas, o a solicitud de la paciente o familia.

\section{REFERENCIAS}

1. De Silva NK, Brandt ML. Disorders of the breast in children and adolescents, Part 2: J Pediatr Adolesc Gynecol 2006;19:415-8.

2. Neintein LS, Atkinson J, Diament M. Prevalence and longitudinal study of breast masses in adolescents. J Adolesc Health 1993;14:277-81. 
3. Duflos C, Plubureau G, Thibaud E, Kuttenn F. Breast disease in adolescents. Sultan C (ed). In: Pediatric and Adolescent Gynecology. Evidence-Based Clinical Practice. Endocr Dev. Basel, Karger, 2004, vol 7, pp 1-8.

4. Hughes LE, Mansel RE, Webster DJ. Aberrations of Normal Development and Involution (ANDI): a new perspective on pathogenesis and nomenclature of benign breast disorders. Lancet 1987;2:1316-9.

5. Fibroadenomas and related tumors. Hughes, Mansel \& Webster's Benign Disorders and Diseases of the Breast. Mansel R (ed). pp 81-107.

6. Greenberg R, Skomick Y, Kaplan O. Management of breast fibroadenomas. J Gen Intern Med 1998;13:6405.

7. Kaneda HJ, Mack J, Kasales C, Schetter S. Pediatric and adolescent breast masses: a review of pathophisiology, imaging, diagnosis and treatment. AJR Am J Roentgenol 2013;200:W204-12.

8. Alle KM, Moss J, Venegas RJ, Khalkhali I, Klein SR. Conservative management of fibroadenoma of the breast. Br J Surg 1996;83:992-3.

9. Greenberg R ,Skornick Y, Kaplan O. Management of breast fibroadenomas J Gen Intern Med 1998;13:6405.

10. Ng WK, Mrad MA, Brown MH. Juvenile fibroadenoma of the breast: Treatment and literature review. Can J Plast Surg 2011 Fall;19:105-7.

11. West KW, Rescoria FJ, Scherer LR III, Grosfeld $\mathrm{JL}$. Diagnosis and treatment of symptomatic breast masses in the pediatric population. J Pediatr Surg 1995;30:182-6.

12. Cant PJ, Madden MV, Coleman MG, Dent DM. Nonoperative management of breast masses diagnosed as fibroadenoma. Br J Surg 1995;82:792-4.

13. Giani C, D'Amore E, Delarue JC, Mouriesse H, MayLevin F, Sancho-Garnier H, Breccia M, Contesso G. Estrogen and progesterone receptors in benign breast tumors and lesions: relationship with histological and cytological features. Int J Cancer 1986;37:7-10.

14. Mauvais-Jarvis P, Kuttenn F, Gompel A. Antiestrogen action of progesterone in breast tissue. Breast Cancer Res Treat 1986;8:179-88

15. Gompel A, Chaouat M,Hugol D,Forgez $P$ Steroidal hormones and proliferation, differentiation and apoptosis in breast cells. . Maturitas. 2004 Sep 24;49(1):1624

16. Kramer B, Seeger $H$, Wallwiener D, Mueck AO. The effect of progesterone, testosterone and synthetic progestogens on growth factor- and estradiol-treated human cancerous and benign breast cells . Eur J Obstet Gynecol Reprod Biol 2006;129:77-83.

17. Courtillot C, Chakhtoura Z, Bogorad R, Genestie C, Bernichtein S, et al; Benign Breast Diseases Study Group. Characterization of two constitutively active prolactin receptor variants in a cohort of 95 women with multiple breast fibroadenomas. J Clin Endocrinol Metab 2010;95:271-9.

18. Coriaty Nelson Z, Ray RM, Gao DL, Thomas DB. Risk factors for fibroadenoma in a cohort of female textile workers in Shanghai, China. Am J Epidemiol 2002;156:599-605.

19. Greenblatt RB, Nezhat C, Ben-Nun I. The treatment of benign breast disease with danazol. Fertil Steril 1980;34:242-5.

20. Cupceancu B. Combined tamoxifen-lynestrol treatment in benign breast disease. Endocrinologie 1985;23:265-72.

21. Smith GE, Burrows P. Ultrasound diagnosis of fibroadenoma. Is biopsy always necessary? Clin Radiol 2008;63:511-5.

22. Sanchez R, Ladino-Torres MF, Bernat JA, Joe A, DiPietro MA. Breast fibroadenomas in the pediatric population: common and uncommon sonographic findings. Pediat Radiol 2010;40:1681-9. 